\title{
Refined empirical stability criterion for nonlinear Schrödinger solitons under spatiotemporal forcing
}

\author{
Franz G. Mertens* \\ Physikalisches Institut, Universität Bayreuth, D-95440 Bayreuth, Germany \\ Niurka R. Quintero ${ }^{\dagger}$ \\ Departamento de Física Aplicada I, E.U.P., Universidad de Sevilla, c/ Virgen de África 7, E-41011 Sevilla, Spain \\ I. V. Barashenkov \\ Department of Mathematics, University of Cape Town, Rondebosch 7701, South Africa
}

A. R. Bishop

Los Alamos National Laboratory, Los Alamos, New Mexico 87545, USA

(Received 31 January 2011; revised manuscript received 28 June 2011; published 25 August 2011)

\begin{abstract}
We investigate the dynamics of traveling oscillating solitons of the cubic nonlinear Schrödinger (NLS) equation under an external spatiotemporal forcing of the form $f(x, t)=a \exp [i K(t) x]$. For the case of time-independent forcing, a stability criterion for these solitons, which is based on a collective coordinate theory, was recently conjectured. We show that the proposed criterion has a limited applicability and present a refined criterion which is generally applicable, as confirmed by direct simulations. This includes more general situations where $K(t)$ is harmonic or biharmonic, with or without a damping term in the NLS equation. The refined criterion states that the soliton will be unstable if the "stability curve" $p(v)$, where $p(t)$ and $v(t)$ are the normalized momentum and the velocity of the soliton, has a section with a negative slope. In the case of a constant $K$ and zero damping, we use the collective coordinate solutions to compute a "phase portrait" of the soliton where its dynamics is represented by two-dimensional projections of its trajectories in the four-dimensional space of collective coordinates. We conjecture, and confirm by simulations, that the soliton is unstable if a section of the resulting closed curve on the portrait has a negative sense of rotation.
\end{abstract}

DOI: 10.1103/PhysRevE.84.026614

PACS number(s): $05.45 . Y v$

\section{INTRODUCTION}

The externally driven nonlinear Schrödinger (NLS) equation arises in a variety of applications, including charge density waves [1], long Josephson junctions [2], optical fibers [3-5], and plasmas driven by rf fields [6]. We use the NLS equation in the form

$$
i u_{t}+u_{x x}+2|u|^{2} u+\delta u=R[u(x, t) ; x, t],
$$

with the perturbation

$$
R=f(x, t)-i \beta u(x, t),
$$

where $f(x, t)$ is a direct (external) driving force and the second term accounts for the dissipation. Different forms of the driving force were considered, e.g., the ac driving, $f=\epsilon \exp (i \omega t)$ $[1,7,8]$, and the driving by a plane wave, $f=\epsilon \exp [i(k x-$ $\omega t)][5,9]$. A more general form $f=\epsilon \exp [i g(x, t)-i \omega t]$, where $g$ is a function of $x-v t$, was also considered, but no localized solutions were discussed [9].

The present paper continues the analysis [10] of the soliton dynamics under the spatiotemporal driving of the form

$$
f(x, t)=a e^{i K(t) x} .
$$

\footnotetext{
*franz.mertens@uni-bayreuth.de

†niurka@us.es
}

A discrete version of Eq. (1) was used to model nonlinear optical waveguide arrays in which discrete cavity solitons can be excited [11]. In that application, $\delta$ is the cavity detuning parameter, and $f(x, t)$ is replaced with $f_{n}(t)=a \exp \left(i \phi_{\text {in }} n\right)$, where $n$ numbers the resonators and $\phi_{\text {in }}(t)$ is the incident angle of the laser pump light. A biharmonic function $\phi_{\text {in }}(t)$ was used in order to generate a ratchet effect [12]. In the present paper, we also obtain a ratchet effect by using a biharmonic driving (Sec. V). This is interesting because there are only a few reports on ratchets with nontopological solitons [12-14]; most of the literature concerns ratchets with topological solitons, e.g., [15-19].

In Ref. [10], Eq. (1) was simulated using the one-soliton solution of the unperturbed NLS equation as the initial condition. The soliton's position, velocity, amplitude, and phase served as parameters of the initial condition (IC).

In the case of zero damping and time-independent, spatially periodic driving of the form $f(x)=\exp (i K x)$, the resulting solitons were observed [10] to display periodic oscillations of their positions, velocities, amplitudes, and phases. Although the driving force has zero spatial average, the soliton's net motion is unidirectional. (This contrasts with the case of a perturbation $V(x) u$ with periodic $V(x)$, where the soliton performs oscillations about a minimum of $V(x)$ [20]).

A large number of sample points in the parameter space $(a, K, \delta)$ were examined by varying the initial amplitude $\eta_{0}$, with the other initial conditions kept fixed. The initial configuration was seen to evolve into a stable soliton only 
when $\eta_{0}$ was taken from one of the "stability windows." For $\eta_{0}$ outside the stability windows, the solitonic initial condition was observed to decay or break into two or more fragments, which would subsequently decay [10].

As a first step toward the understanding of the observed dynamics of solitons, the authors of Ref. [10] proposed an empirical stability criterion based on a collective coordinate (CC) description. The collective coordinate analysis produces a set of coupled nonlinear ordinary differential equations (ODEs) for the soliton's position $q$, amplitude $\eta$, normalized momentum $p$, and phase $\Phi$. An approximate solution of this dynamical system is given by trigonometric functions and can be obtained explicitly, except when the initial condition $\eta_{0}$ is chosen near one of the stability boundaries. In the latter case, the collective coordinate equations had to be analyzed numerically and their solutions were found to be highly anharmonic.

We have positively tested the predictions of the proposed stability criterion by simulations [numerical solutions of the full NLS Eq. (1)] for many classes of initial conditions. However, the tests have turned out to be negative when the initial momentum was too large, i.e., $p_{0}>K$ for positive $K$ (or $p_{0}<K$ for negative $K$ ). In this paper, we therefore propose a refined stability criterion, which, as we show, makes correct predictions not only for the case $K=$ constant (with all classes of initial conditions), but also for harmonic and biharmonic $K(t)$. The new criterion is a sufficient condition, which states that the soliton will be unstable in simulations if the "stability curve" $p(v)$ has a branch with a negative slope. This curve is obtained as a parametric plot of the normalized momentum of the soliton,

$$
p=\frac{P(t)}{N(t)}
$$

versus its velocity

$$
v=\dot{q}(t) \text {. }
$$

Here $P=4 \eta p$ is the canonical momentum of the soliton, and $N=4 \eta$ is the norm which is canonically conjugated to the soliton's phase $\Phi(t)$; see Sec. II. In the original criterion [10], the stability curve was defined as $P(v)$. At the end of Sec. III, we present an example which demonstrates by an anatical calculation that the normalized momentum $p$, instead of the canonical momentum $P$, has to be used in the stability criterion.

It is important to emphasize that the soliton's stability or instability is judged not on the basis of the stability of solutions to the collective coordinate equations. (The latter are stable in most cases). The soliton's stability is rather decided on the basis of some of its properties, which are captured by the $p(v)$ curve of the corresponding collective coordinate solutions. The proposed empirical criterion reproduces the numerically observed positions of the stability windows to an accuracy of better than $1 \%$, despite the complexity of the stability diagram in the parameter space [10].

The stability criteria for the homogeneous (translation invariant) NLS equation available in the literature are restricted to (a) bright solitons, i.e., solutions decaying to zero at the spatial infinities, with time dependencies of the form $e^{i \Lambda t}$ (and those reducible to this form by a Galileian transformation); and (b) traveling dark solitons, i.e., solutions approaching nonzero constant values as $x \rightarrow \pm \infty$. The criteria are insensitive to the particular form of the nonlinearity as long as it is conservative and $U(1)$ invariant, i.e., as long as the equation does not include any damping or driving terms.

In the case of the bright solitons of the form $u(x, t)=$ $u_{s}(x) e^{i \Lambda t}$, the Vakhitov-Kolokolov criterion states that if the corresponding energy Hessian has only one negative eigenvalue, then the soliton is stable if $d N / d \Lambda>0$ and unstable otherwise [21-23]. Here $N=\int|u|^{2} d x$; depending on the physical context, $N$ is referred to as the number of particles contained in the soliton or the total power of the optical beam. (See also [24] for the Hamiltonian-versus-number of particles formulation of this criterion.)

In the case of dark solitons of the form $u(x, t)=u(x-$ $\tilde{V} t)$, with $|u|^{2} \rightarrow \rho_{0}$ as $|x| \rightarrow \infty$, a similar criterion [25-28] involves the (renormalized) field momentum

$$
\tilde{P}=\frac{i}{2} \int\left(u_{x}^{*} u-u_{x} u^{*}\right)\left(1-\frac{\rho_{0}}{|u|^{2}}\right) d x .
$$

The dark soliton traveling at the constant velocity $\tilde{V}$ is stable if $d \tilde{P} / d \tilde{V}<0$, and unstable otherwise.

Some parts of the stability analysis of the traveling dark solitons [28] can be carried over to the case of the traveling solitons of the NLS equation with a driving term. Namely, one can show [29] that a pair of linearized eigenvalues crosses from the imaginary to real axis at the value $\tilde{V}$ where $d \tilde{P} / d \tilde{V}=$ 0 . The sign of the derivative $d \tilde{P} / d \tilde{V}$ required for stability depends on the type of the soliton; some classes of solitons require $d \tilde{P} / d \tilde{V}<0$, whereas other classes are stable when $d \tilde{P} / d \tilde{V}>0$. (An additional complication is the presence of oscillatory instabilities where two eigenvalues collide on the imaginary axis and acquire opposite real parts. The oscillatory instabilities do not affect the sign of $d \tilde{P} / d \tilde{V}$.)

In these analyses, each point of the curve $\tilde{P}(\tilde{V})$ represents a soliton traveling at a particular constant velocity $\tilde{V}$; therefore the curve is a characteristic of the whole family of solitons. The values of $\tilde{V}$ where $d \tilde{P} / d \tilde{V}=0$ break the family into parts with different stability properties. In contrast to this, each oscillatory solution of the collective coordinate equations [10] has its own, individual $p(v)$ curve, the whole of which is traced periodically in time. The shape of this curve determines whether the corresponding soliton is stable or not.

The present paper has several goals: First, we propose a refined stability criterion. Second, we study the internal structure of the instability regions. We will demonstrate that these regions consist of subregions characterized by instabilities of different types. The existence of the subregions will be predicted by the analysis of the reduced dynamical system and confirmed by direct simulations of the full partial differential equation (PDE) (Sec. III). In obtaining the reduced dynamical system, we modify the original collective coordinate approach of Ref. [10] (Sec. II). In addition to producing bounded trajectories (a property essential for the stability analysis), the modified approach provides a much easier derivation of the canonical soliton momentum and the Hamilton function in terms of the canonical variables (Sec. II).

Third, we demonstrate that a certain "phase portrait" of the soliton on the complex plane can be used as an alternative stability diagnostic (Sec. III). However, the phase portrait 
requires the phase of the soliton to be periodic in time. This can be achieved by the above-mentioned modification of the original collective coordinate approach [10] in which the phase was not periodic, in contrast to the other three collective coordinates.

Finally, we explore the applicability of our refined stability criterion to inhomogeneous forcings of the form $f(x, t)=$ $a \exp [i K(t) x]$ in Eq. (3). We will start with a harmonically oscillating $K(t)$, with and without the damping term in the right-hand side of (1) (Sec. IV). After that, in Sec. V, we will consider a biharmonic $K(t)$ with a broken temporal symmetry. (The temporal symmetry breaking will accompany the breaking of the spatial symmetry by the inhomogeneous driving.)

\section{MODIFIED COLLECTIVE COORDINATE THEORY}

The one-soliton solution of the unperturbed NLS equation is given by [30]

$$
u(x, t)=2 i \eta \operatorname{sech}[2 \eta(x-\zeta)] e^{-i(2 \xi x+\phi)},
$$

where $\eta$ and $\xi$ are real parameters $(\eta>0) ; \zeta=\zeta_{0}-4 \xi t$ gives the coordinate of the soliton's center, and $\phi=\phi_{0}+\left(4 \xi^{2}-\right.$ $\left.4 \eta^{2}-\delta\right) t$ is the soliton's phase. The collective coordinate theory of Ref. [10] assumed that for sufficiently small perturbations $R$ in Eq. (2), the soliton shape and dynamics can be described, approximately, by Eq. (7), where $\eta(t), \xi(t)$, $\zeta(t)$, and $\phi(t)$ are functions of time.

We now show that the following modification of this ansatz $[31,32]$ provides a considerable improvement of the collective coordinate theory of Ref. [10]:

$$
u(x, t)=2 i \eta \operatorname{sech}[2 \eta(x-q)] e^{i[p(x-q)-\Phi]} .
$$

Equation (8) is obtained from (7) by setting $-2 \xi=p, \zeta=q$, and $\phi=\Phi-2 \xi \zeta=\Phi+p q$. Here only the last replacement is essential for the above-mentioned improvement of the collective coordinate theory. The four collective coordinate equations of Ref. [10] are replaced with

$$
\begin{gathered}
\dot{\eta}=-2 \beta \eta-\frac{\pi}{2} a \operatorname{sech} A \cos B, \\
\dot{q}=2 p+\frac{\pi^{2}}{8} \frac{a}{\eta^{2}} \operatorname{sech} A \tanh A \sin B, \\
\dot{p}=-2 a A \operatorname{sech} A \cos B, \\
\dot{\Phi}+p \dot{q}=p^{2}-4 \eta^{2}-\delta+\frac{\pi}{2} \frac{a A}{\eta} \operatorname{sech} A \tanh A \sin B,
\end{gathered}
$$

with

$$
\begin{gathered}
A(t)=\frac{\pi}{4 \eta(t)}[K(t)-p(t)], \\
B(t)=\Phi(t)+K(t) q(t) .
\end{gathered}
$$

The new formulation has the following advantages.

(i) Consider the Lagrangian for Eqs. (9)-(12),

$$
L=4 \eta \dot{\Phi}+4 \eta p \dot{q}-4 \eta p^{2}+\frac{16}{3} \eta^{3}+4 \delta \eta-2 \pi a \operatorname{sech} A \sin B .
$$

The momentum conjugate to the phase $\Phi$ is

$$
\frac{\partial L}{\partial \dot{\Phi}}=4 \eta,
$$

which is equal to the norm $\left(\int|u|^{2} d x\right)$ of the waveform (8). The momentum conjugate to the soliton's position is

$$
\frac{\partial L}{\partial \dot{q}}=4 \eta p .
$$

The advantage of the new formulation is that this is equal to the field momentum of the configuration (8),

$$
P=\frac{i}{2} \int_{-\infty}^{+\infty}\left(u_{x}^{*} u-u_{x} u^{*}\right) d x,
$$

whereas in Ref. [10] the second canonical momentum was defined by $\partial L / \partial \dot{p}=-4 \eta q$, which did not have any obvious physical interpretation.

If the dissipative term $-i \beta u$ in (2) has a nonzero coefficient, we have to use the generalized Euler-Lagrange formalism with the dissipation function

$$
F=i \beta \int_{-\infty}^{+\infty}\left(u u_{t}^{*}-u^{*} u_{t}\right) d x .
$$

Substituting (8) in (19), we obtain

$$
F=-8 \beta \eta(\dot{\Phi}+p \dot{q}) .
$$

The generalized Euler-Lagrange equations are

$$
\frac{d}{d t} \frac{\partial L}{\partial \dot{\Psi}}-\frac{\partial L}{\partial \Psi}=\frac{\partial F}{\partial \dot{\Psi}},
$$

where $\Psi$ represents each of the four collective coordinates $\eta$, $q, p$, and $\Phi$.

(ii) Since $P=4 \eta p$ is the canonically conjugate momentum for $q$, the Legendre transform to the canonical Hamiltonian is easily performed: $H=N \dot{\Phi}+P \dot{q}-L$. This gives

$$
H=\frac{1}{N} P^{2}-\frac{1}{12} N^{3}-\delta N+2 \pi a \operatorname{sech} A \sin B .
$$

In Ref. [10], this Hamiltonian could only be obtained via a canonical transformation.

When the forcing $f(x)=a e^{i K x}$ is time independent and damping $\beta=0$, the collective coordinates $\eta$ and $p$ perform periodic oscillations, whereas $q(t)$ and $\Phi(t)$ are given by periodic functions superimposed over linearly growing functions of $t$. In contrast to this, the variable $\phi=\Phi+p q$ used in Ref. [10] will obviously exhibit oscillations with a (linearly) growing amplitude.

\section{TIME-INDEPENDENT, SPATIALLY PERIODIC FORCE}

Our approach consists in the numerical solution of the collective coordinate equations (9)-(12) for representative values of the parameters and the initial conditions $\eta_{0}, q_{0}$, $p_{0}$, and $\Phi_{0}$. Each collective coordinate orbit is then used to compute $p(t)$ and $v(t)$ in Eqs. (4) and (5), and to plot $p$ against $v$. If some part of this "stability curve" has a negative slope, we predict that the soliton will become unstable in simulations of the PDE (1) starting with the initial condition (8), with the same $\eta_{0}, p_{0}, q_{0}$, and $\Phi_{0}$.

Since the collective coordinate approximation can work only for small perturbations, we choose a small driving amplitude $a=0.05$ in $f(x)=a e^{i K x}$. We also take $K=0.1$, which means that the spatial period of the forcing $L=2 \pi / K \gg 1$. We are interested in periodic solutions and, therefore, we set 

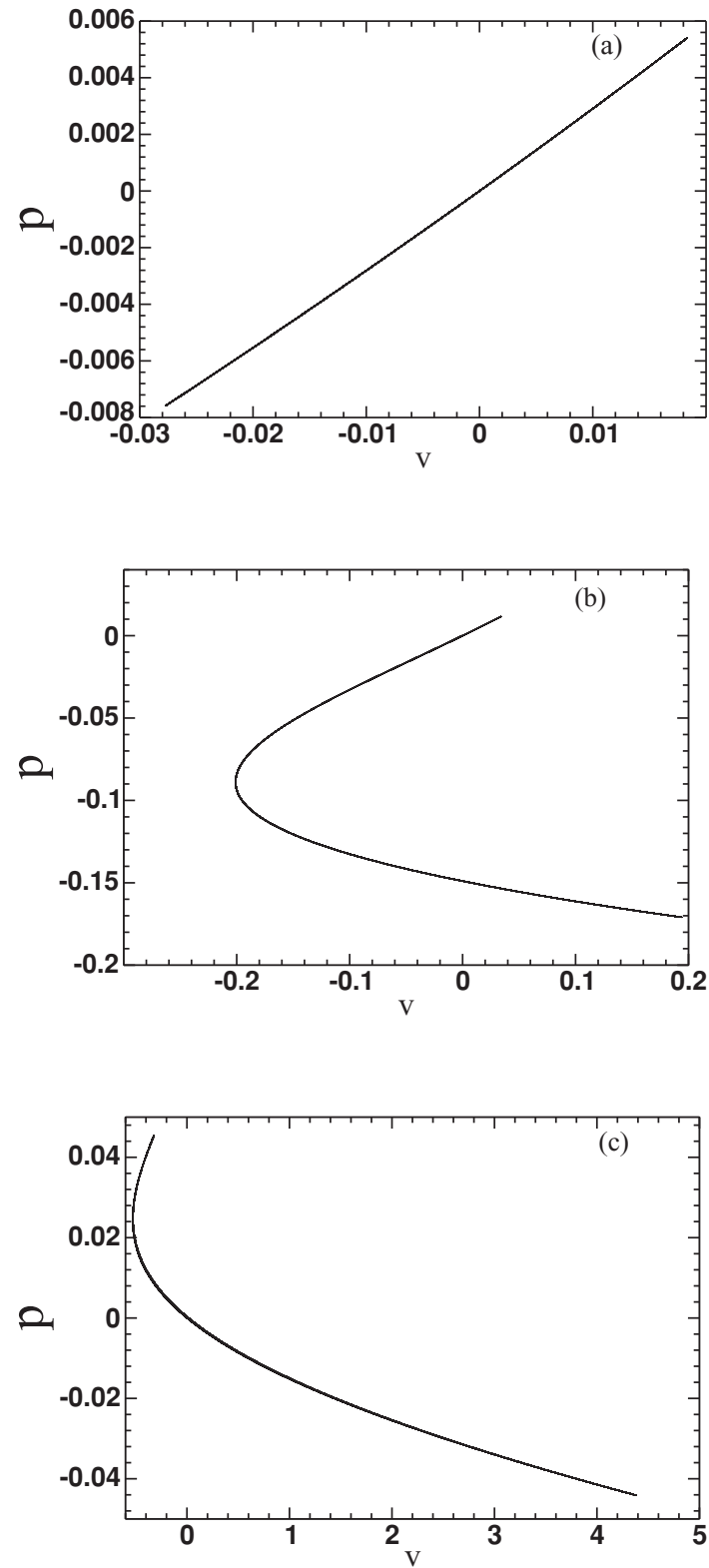

FIG. 1. Stability curve $p(v)$ corresponding to $a=0.05, K=0.1$, $\delta=-1$, and $\beta=0$. The initial conditions for the Eqs. (9)-(12) were $q_{0}=p_{0}=\Phi_{0}=0$ and (a) $\eta_{0}=0.8$, (b) $\eta_{0}=0.65$, (c) $\eta_{0}=0.1$. The integration time $t_{f}=1000$.

the damping parameter $\beta=0$. Damped oscillatory solutions were already considered in Ref. [10].

For $\delta \geqslant 0$, the $p(v)$ curve predicts only unstable solitons, which is confirmed by simulations. For $\delta<0$, there are typically several stability regions, which grow as $|\delta|$ is increased, while the parameters $a$ and $K$ are fixed [10]. We concentrate here on the simplest case with only one stability and one instability region. Namely, we choose $\delta=-1, q_{0}=$ $p_{0}=\Phi_{0}=0$ for which the soliton solutions are predicted to be stable if $\eta_{0} \geqslant \eta_{c}^{(1)}=0.684$ [Fig. 1(a)] and unstable for $\eta_{0}<\eta_{c}^{(1)}$. This is confirmed by our simulations of the PDE (1) to an accuracy of better than $1 \%$ in $\eta_{c}^{(1)}$.

The range of initial amplitudes $\eta_{0}$, for which the solutions of Eqs. (9)-(12) feature a $p(v)$ curve with a descending branch, can be divided into two subintervals, $0<\eta_{0}<\eta_{c}^{(2)}$ and $\eta_{c}^{(2)}<\eta_{0}<\eta_{c}^{(1)}$, where $\eta_{c}^{(2)}=0.288$. In the upper subinterval, $\left(\eta_{c}^{(2)}, \eta_{c}^{(1)}\right)$, the curve $p(v)$ exhibits two long branches, one with a positive and the other with a negative slope [Fig. 1(b)]. In the lower subinterval, the positive-slope branch is short and very steep [Fig. 1(c)]. The difference in the shape of the stability curves suggests different types of instability in the two subregions; however, the way in which the instabilities are different cannot be deduced from the $p(v)$ curve alone. To gain further insight into this difference, we plot a phase portrait for the dynamical system (9)-(12). The vertical and horizontal axes in the portrait are chosen so that they admit a clear interpretation in terms of the full PDE, Eq. (1). To this end, we first transform to the frame of reference moving with the velocity $V_{f}$ :

$$
u(x, t)=\Psi(X, t) e^{i K x}, \quad X=x-V_{f} t,
$$

where $V_{f}=2 K$. Equation (1) is taken to an NLS equation driven by a space-time independent external force:

$$
i \Psi_{t}+\Psi_{X X}+2|\Psi|^{2} \Psi+\left(\delta-K^{2}\right) \Psi=a .
$$

[This equation was previously studied in a different context $[7,8,33,34]$ and two static soliton solutions were obtained explicitly [34]. Unlike [7,8,33,34], we focus here on moving solitons of Eq. (24).]

Under the transformation (23), the collective coordinate ansatz (8) becomes

$$
\Psi(X, t)=2 i \eta \operatorname{sech}\left[2 \eta\left(X+V_{f} t-q\right)\right] e^{-i\left[(K-p)\left(X+V_{f} t\right)+p q+\Phi\right]} .
$$

The $\eta(t)$ and $p(t)$ components of the oscillatory solutions of (9)-(12) are periodic with period $T$, whereas $q$ and $\Phi$ are of the form $q(t)=\bar{v} t+q^{(p)}(t), \Phi(t)=-\alpha t+\Phi^{(p)}(t)$, where $q^{(p)}(t)$ and $\Phi^{(p)}(t)$ are $T$-periodic functions and $\alpha$ is a constant [10]. The corresponding soliton given by (8) and (25) has the mean velocity $\bar{v}$ in the original frame of reference, and $\bar{v}-V_{f}$ in the moving frame.

At the point $x=\bar{v} t$ [or, equivalently, at $X=\left(\bar{v}-V_{f}\right) t$, the function (25) has the following time dependence:

$$
\Psi=2 i \eta \operatorname{sech}[2 \eta(\bar{v} t-q)] e^{-i[K \bar{v} t-p(\bar{v} t-q)+\Phi]} .
$$

The function (26) is a collective coordinate counterpart of the $\Psi$ field at the center of the soliton solution of Eq. (24). Comparing Eq. (26) to the function $\left.\Psi(X, t)\right|_{X=\bar{v} t-V_{f} t}$ obtained in the direct numerical simulations of the full PDE (1), one can assess the validity and accuracy of the collective coordinate approximation. For this reason, we choose the complex function (26) as a representative of the four-dimensional dynamics, and plot its real versus imaginary part to generate the corresponding phase portrait. The soliton dynamics is described by the resulting orbits of the phase portrait.

One can readily verify that these orbits are closed. Indeed, the modulus of the function (26) is periodic with period $T$. Therefore, to demonstrate the closure, one just needs to show that the argument of $\Psi$ changes by an integer multiple of $2 \pi$ over the period. We have $\arg \Psi=(\alpha-K \bar{v}) t-p^{(p)} q^{(p)}-$ $\Phi^{(p)}$, where the last two terms are $T$ periodic. As for the first term, the constants $\bar{v}$ and $\alpha$ are found as the coefficients of the linearly growing components of $q(t)$ and $\Phi(t)$, respectively. 


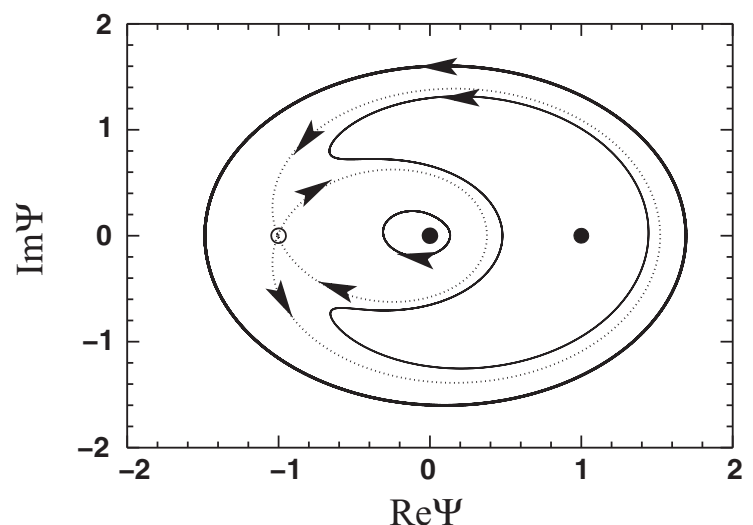

FIG. 2. The phase portrait of the system (9)-(12) with $a, K, \delta$, and $\beta$ as in Fig. 1. Shown is $\operatorname{Im} \Psi\left(X=\bar{v} t-V_{f} t, t\right)$ vs. $\operatorname{Re} \Psi(X=$ $\left.\bar{v} t-V_{f} t, t\right)$. The large ellipse corresponds to $\eta_{0}=0.8$, the horseshoe to $\eta_{0}=0.65$, and the small ellipse to $\eta_{0}=0.1$. Other initial conditions are as in Fig. 1. The separatrix is shown by the dotted curve. The filled and open circles are stable and unstable fixed points, respectively.

The numerical solution of Eqs. (9)-(12) verifies $\alpha-K \bar{v}=$ $2 \pi / T$ in the stability range $\eta_{0} \geqslant \eta_{c}^{(1)} ; \alpha-K \bar{v}=-2 \pi / T$ in the lower instability subinterval $0<\eta_{0}<\eta_{c}^{(2)}$; and $K \bar{v}-\alpha=$ 0 in the upper instability subinterval $\eta_{c}^{(2)}<\eta_{0}<\eta_{c}^{(1)}$. These relations between $\bar{v}$ and $\alpha$ hold to a numerical accuracy of $O\left(10^{-5}\right)$.

Trajectories resulting from initial conditions in the interval $\eta_{0}>\eta_{c}^{(1)}$ are ellipses, with a positive sense of rotation (Fig. 2). The ellipses enclose a stable and an unstable fixed point on the real axis at about +1 and -1 , respectively. (For the definition and calculation of these points, see the Appendix). Figure 3(a) compares the soliton amplitude $\eta(t)$ from the collective coordinate theory to the amplitude measured in the direct simulations of Eq. (1).

For the upper instability subinterval $\eta_{c}^{(2)}<\eta_{0}<\eta_{c}^{(1)}$, the phase trajectory is a horseshoe (Fig. 2). This curve consists of an outer part with a positive sense of rotation and an inner part with a negative sense of rotation relative to the origin. The two parts are correlated with the two branches with positive and negative slopes, respectively, of the $p(v)$ curve in Fig. 1(b). The soliton instability is seen in the simulation result in Fig. 3(b). Note that the first harmonic vanishes after about 30 time units, while the second harmonic persists. Eventually the soliton decays: the amplitude approaches zero while the width tends to infinity.

For the lower instability interval $0<\eta_{0}<\eta_{c}^{(2)}$, the situation is quite different, both in the collective coordinate theory and in the simulations: The phase portrait features an ellipse, but with the negative sense of rotation (Fig. 2). Moreover, the ellipse is much smaller than the one arising in the stability region so that it encloses only one fixed point. This fits with the simulations in which the soliton remains metastable for a relatively long time, exhibiting a periodic modulation of the oscillation amplitude [Fig. 3(c)], but then the instability sets in [Fig. 3(d)].

So far we have varied $\eta_{0}$, with $p_{0}=q_{0}=\Phi_{0}=0 \mathrm{kept}$ fixed. We now consider the stability diagram in the $\eta_{0}-p_{0}$ plane near the critical value $\eta_{c}^{(1)}$, which separates the stability and the
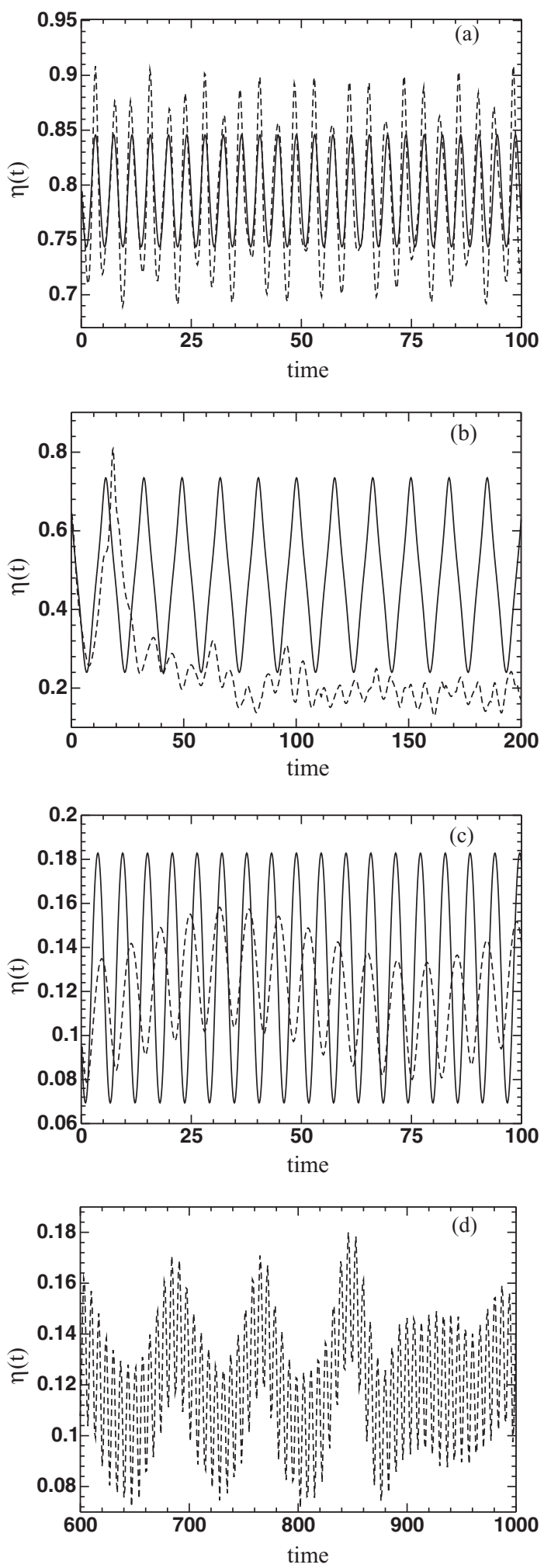

FIG. 3. Soliton amplitude $\eta(t)$ from the collective coordinate theory (solid lines) and from the simulations (dashed lines). The parameters and the initial conditions are the same as in Fig. 1. (a) $\eta_{0}=0.8$, (b) $\eta_{0}=0.65$, (c) $\eta_{0}=0.1$ (shown are results for early times $0 \leqslant t \leqslant 100$ ); (d) $\eta_{0}=0.1$ (shown are simulation results for late times $600 \leqslant t \leqslant 1000$.)

upper instability interval. Figure 4 shows that a nonzero value of the normalized momentum $p_{0}$ has a stabilizing effect on the 


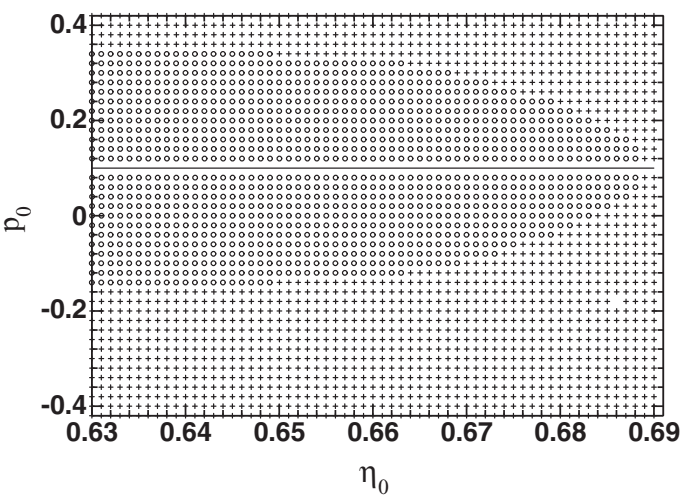

FIG. 4. Stability diagram near $\eta_{c}^{(1)}=0.684$, with $q_{0}=\Phi_{0}=0$. Parameters: $a=0.05, K=0.1, \delta=-1, \beta=0$. Circles: unstable soliton. Pluses: stable soliton. The horizontal line marks $p_{0}=K$. For this value of $p_{0}$, the $p(v)$ curve collapses into a point.

soliton; including nonzero $p_{0}$ enlarges the stability domain. The curve which separates the stability and instability regions is roughly a parabola.

Finally we would like to emphasize the crucial role played by the normalized momentum $p$ in the stability analysis - rather than by the canonical momentum $P$ (as was suggested in [10]). We have established that the empirical stability criterion proposed in [10] disagrees with the results of numerical simulations when the initial normalized momentum is too large, i.e., $p_{0}>K$ (for positive $K$ ). Let, for instance, the parameters of the equation take the same values as in Fig. $1(a=0.05, K=0.1, \delta=-1$, and $\beta=0)$, and take the same initial conditions as for the stable stationary solution in the Appendix $\left(\eta_{0}=0.5 \sqrt{K^{2}-\delta}, \Phi_{0}=\pi / 2\right.$, $q_{0}=0$ ), except that now $p_{0}=K+d$, with $0<d<0.2$. Here the numerical solutions of the collective coordinate equations can be very well approximated by $p(t)=p_{0}+$ $a_{p}(1-\cos \Omega t)$ and $v(t)=v_{0}+a_{v}(1-\cos \Omega t)$, where $p_{0}, v_{0}$, $a_{p}$, and $a_{v}>0$. Thus $p(v)$ is a straight line with the slope $a_{p} / a_{v}>0$. This predicts stability, and so does the orbit in the phase portrait which is a small ellipse with a positive sense of rotation around the stable fixed point at about +1 on the real axis. The stability is confirmed by simulations. However, when the momentum $P=4 \eta p$ is used, the situation is different. This time, $\eta(t)$ can be expressed via $p(t)$ by using the exact relation $\eta=\eta_{0}\left(p_{0}-K\right) /(p-K),(p \neq K)$, which is obtained from Eqs. (9) and (11) where one integration has been carried out. Substituting the above expression for $p(v)$, one can check that $P$ decreases when $v$ increases, and vice versa. Thus the slope $d P / d v<0$ predicts instability, which disagrees with the simulations.

\section{HARMONIC $K(t)$}

As stated in Sec. I, one of the aims of this paper is to verify whether the stability criterion $p^{\prime}(v)>0$ remains applicable to time-dependent forces of the form $f(x, t)=a e^{i K(t) x}$. In this section, we consider the case of a harmonically modulated forcing wave number,

$$
K(t)=k \sin (\omega t+\theta),
$$
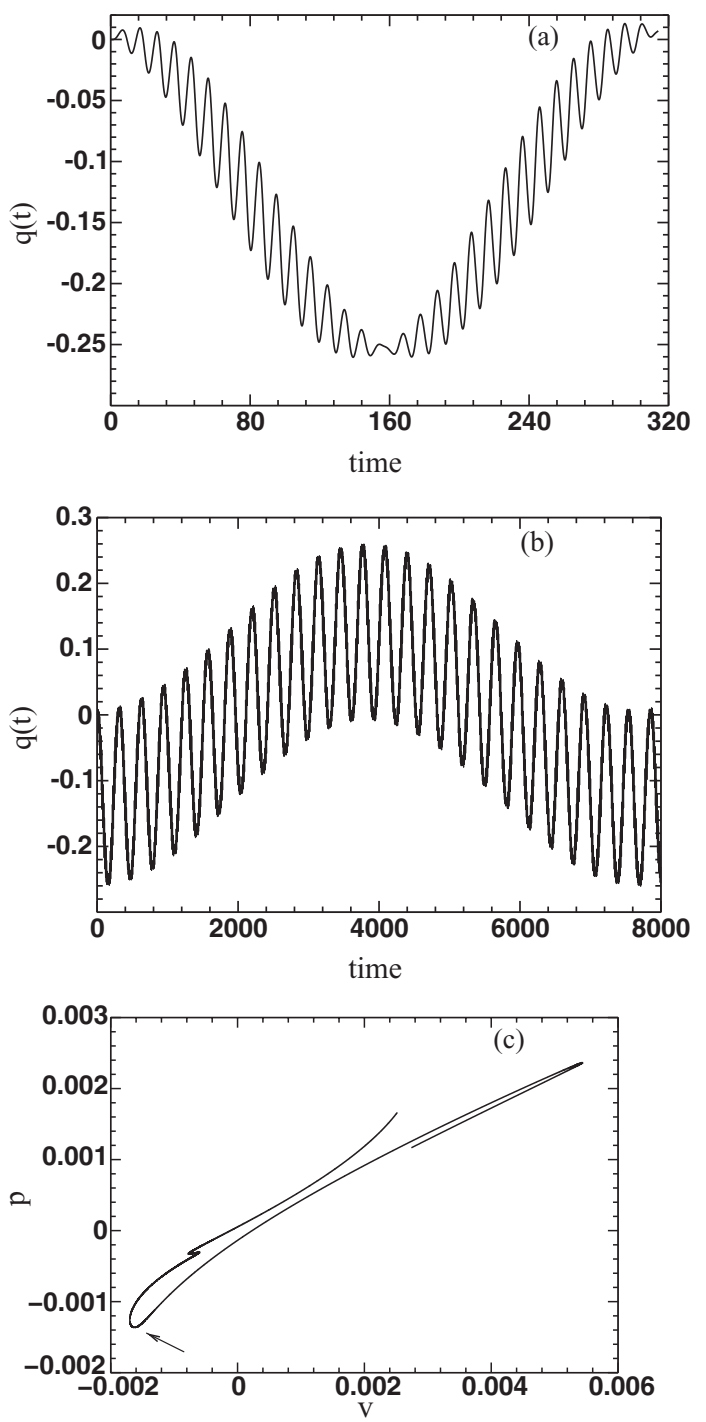

FIG. 5. Collective coordinate results for harmonic $K(t)$, with no damping. $a=0.05, k=-0.1, \omega=0.02, \theta=0, \delta=-3, \beta=0$, $q_{0}=p_{0}=0, \Phi_{0}=\pi / 2, \eta_{0}=1$. (a) $q(t)$ exhibits $\omega_{i}$ oscillations modulated by the frequency $\omega$. Shown is the interval $0 \leqslant t \leqslant$ $T_{d}=2 \pi / \omega$. (b) $q(t)$ exhibits $\omega$ oscillations modulated by the frequency $\omega_{l}=2 \pi / T_{l}$. Here $0 \leqslant t \leqslant 8000$. (c) Stability curve $p(v)$ for $T_{d} / 2-5 \leqslant t \leqslant T_{d} / 2+10$. The arrow points to the section of the curve with a negative slope loop.

first without a damping term in the NLS equation $(\beta=0)$, then with the damping term $(\beta>0)$.

We choose the same parameters as in Sec. III: $a=0.05, k=$ -0.1 , which implies $|K| \ll 1$. In order to be in the adiabatic regime, we choose a small modulation frequency $\omega=0.02$. Finally, we let $\theta=0$ and choose initial conditions $q_{0}=p_{0}=$ $0, \eta_{0}=1$, and $\Phi_{0}=\pi / 2$.

The numerical solutions of the collective coordinate equations (9)-(12) exhibit oscillations with three very different frequencies in their spectrum. This is most explicit in the behavior of $q(t)$ [Figs. 5(a) and 5(b)]. First, there are intrinsic oscillations with the frequency $\omega_{i}$; these have a period $T_{i}$ of the order of 10 , which is similar to the oscillations in the case of the constant $K$ discussed in the previous section. Second, there 
are oscillations with the driving frequency $\omega$ whose period $T_{d}=2 \pi / \omega \approx 314$. Finally, there are oscillations with a very low frequency $\omega_{l}$ and very long period $T_{l} \approx 8000$ [Fig. 5(b)]. The resulting stability curve $p(v)$ exhibits many small loops, which have a short section with a negative slope. An example is given in Fig. 5(c). (For clarity, the curve is plotted only over a short time interval). The negative slope predicts instability; this is confirmed by our simulations of the full PDE.

Stable solitons can be obtained by changing $\eta_{0}$ in such a way that the loops do not arise. This is achieved by suppressing the intrinsic oscillations, since their period $T_{i} \approx 10$ is of the same order as the time scale of the loops; see Fig. 5(c). The intrinsic oscillations disappear when we choose $\eta_{0}=\sqrt{-\delta} / 2$ [Figs. 6(a) and 6(b)]. In this case, $\eta(t)$ performs very small oscillations around $\eta_{0}$ and the two dominant terms on the right-hand side of Eq. (12), namely $-4 \eta_{0}^{2}$ and $-\delta$, cancel each other. Figure 6(c) demonstrates that the small loops have indeed disappeared.

The resolution of Fig. 6(c) does not allow verification of whether there are sections with negative slope near the turning points of the stability curve. We now show that there cannot be any, as the curve develops cusps at the turning points. Consider the region around one of the maxima (or minima) of the $\omega$ oscillations of the collective coordinates [Fig. 6(a)]. The functions $q(t), \eta(t)$, etc. are not symmetric with respect to $t_{m}$ (position of the extremum) due to the existence of the very slow $\omega_{l}$ oscillations. The same holds for $p$; hence,

$$
p(t)= \begin{cases}p_{m}-C_{l}\left(t-t_{m}\right)^{2} & \text { for } t \leqslant t_{m}, \\ p_{m}-C_{r}\left(t-t_{m}\right)^{2} & \text { for } t \geqslant t_{m},\end{cases}
$$

with $C_{l} \neq C_{r}$. For the velocity $v(t)=\dot{q}(t)$, the asymmetry is negligible, compared to the asymmetry of $p(t)$, because the time derivative $\dot{q}$ contains a factor $\omega_{l} \ll 1$. Thus $v(t)=$ $v_{m}-b\left(t-t_{m}\right)^{2}$ for both $t \leqslant t_{m}$ and $t \geqslant t_{m}$. By eliminating $t$, we obtain $p=p_{m}-C_{l}\left(v_{m}-v\right) / b$ as $v$ increases up to its maximal value $v_{m}$, and $v=v_{m}-C_{r}\left(v_{m}-v\right) / b$ as $v$ decreases from $v_{m}$. Thus the stability curve $p(v)$ has a cusp with two different (but positive) slopes, $C_{l} / b$ and $C_{r} / b$, at the turning point $v=v_{m}$. The absence of segments of the curve with $d p / d v<0$ predicts stability for the soliton. This is confirmed by the simulations of the PDE (1).

When the damping term $-i \beta u$ is included in the righthand side of the NLS equation (1), the collective coordinate dynamics simplifies. Namely, both the intrinsic oscillations and the low-frequency oscillations are damped out from solutions of the collective coordinate equations after a transient time $t_{\text {tr }}=1 / \beta$. After this transient, all collective coordinate oscillations become locked to the driving frequency $\omega$. The stability curve in this case consists of two nearly straight lines, which form sharp cusps at both ends [Fig. 7(a)]. Thus there are no sections with a negative slope and the soliton is predicted to be stable. This is confirmed by the simulations of the PDE. For long times $\left(t \gg t_{\mathrm{tr}}\right)$, the average soliton velocity $\bar{v}$ slowly approaches zero [Fig. 7(b)]; this behavior is independent of the initial conditions. Thus there is no unidirectional motion of the soliton for long times; the reason will be established in the next section.

As $\beta$ is decreased, the stability curve becomes wider and the decay of $\bar{v}$ to zero faster. On the contrary, as $\beta$ is increased, the
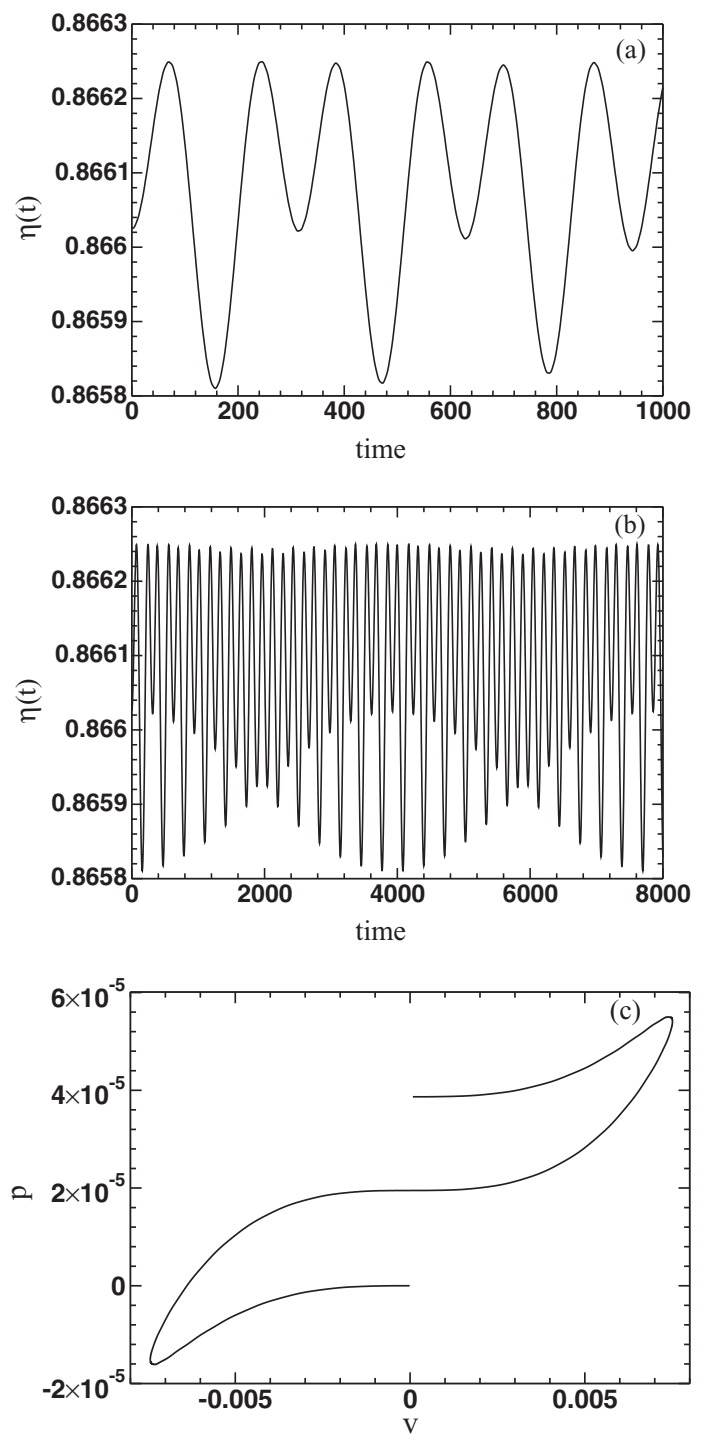

FIG. 6. Collective coordinate results for harmonic $K(t)$, with no damping. Same parameters and initial conditions as in Fig. 5, but $\eta_{0}=\sqrt{-\delta} / 2$. (For this choice, the intrinsic $\omega_{i}$ oscillations vanish.) (a) The amplitude $\eta(t)$ exhibits no $\omega_{i}$ oscillations, only the $\omega$ oscillations modulated by the $\omega_{l}$ oscillations, which are hardly visible for $0 \leqslant t \leqslant$ 1000. (b) In the interval $0 \leqslant t \leqslant 8000$, both the $\omega$ and $\omega_{l}$ oscillations are observed. (c) Stability curve for $0 \leqslant t \leqslant T_{d}$.

stability curve becomes narrower, and the decay of $\bar{v}$ to zero slower. However, for $\beta$ above a critical value $\beta_{c}\left(\beta_{c} \approx 0.035\right.$ for the parameters of Fig. 7), the collective coordinate solutions become unstable. Direct simulations confirm the soliton's instability.

\section{BIHARMONIC DRIVING: RATCHETS}

The simplest ratchet models consider a pointlike particle in a periodic potential driven by an ac force, $f(t)$. Under certain conditions related to the breaking of symmetries, unidirectional motion of the particle can take place despite the applied force having a zero temporal average [35-39]. Particle ratchets were generalized to nonlinear field theoretic systems, in which particles are replaced by solitons [40-46]. 

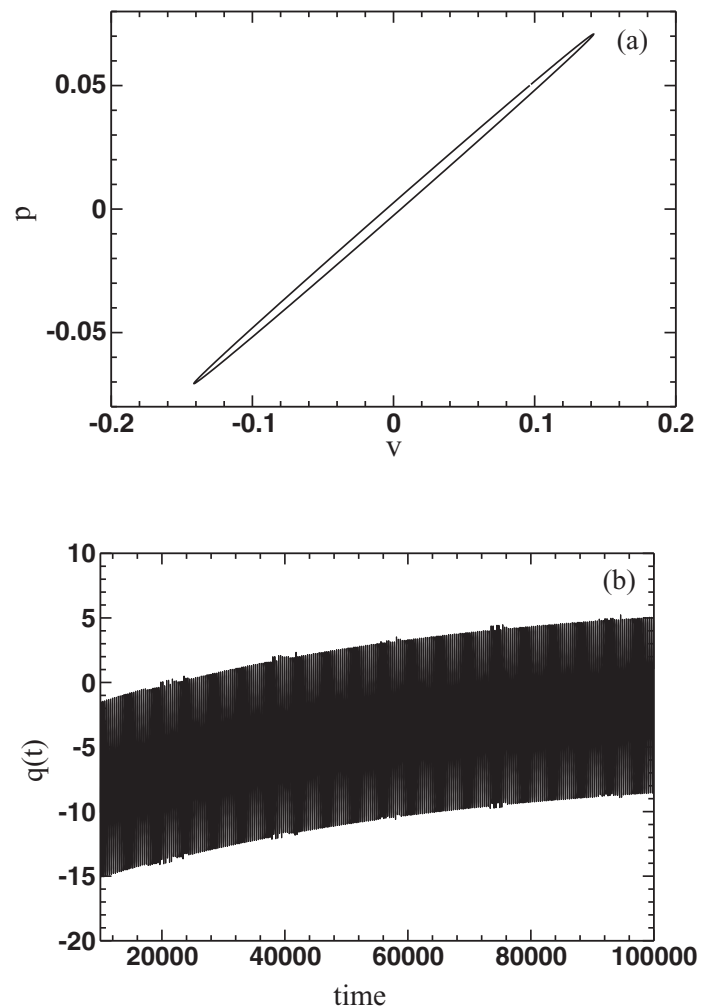

FIG. 7. Collective coordinate results for harmonic $K(t)$, with nonzero damping. The same data is used as in Fig. 6, but $\eta_{0}=0.866 \approx$ $\sqrt{-\delta} / 2, \beta=0.01$. (a) Stability curve $p(v)$ for $3 T_{d} \leqslant t \leqslant 4 T_{d}$. (b) $q(t)$ for $10000 \leqslant t \leqslant 100000$.

In particular, solitons in nonlinear Klein-Gordon systems can move on the average in one direction, if either a temporal or a spatial symmetry is broken.

A temporal symmetry, namely, a time-shift symmetry, is broken by a biharmonic force [47,48]. In this case, the mechanism of the ratchet effect was clarified by a collective coordinate theory employing the soliton position and width as collective coordinates $[15,16,49]$. Due to the coupling between the translational and internal degrees of freedom, energy is pumped nonuniformly into the system, generating a unidirectional motion. The breaking of the time-shift symmetry gives rise to a resonance mechanism that is present whenever the soliton oscillation spectrum comprises at least one of the frequency components of the driving force.

In this section, we investigate whether the NLS solitons show a behavior similar to the Klein-Gordon kinks. Our motivation here stems from the fact that the NLS solitons are nontopological, whereas the vast majority of reports on soliton ratchets have so far focused on topological solitons.

We consider the NLS equation (1) where the perturbation

$$
R=f(x, t)-i \beta u
$$

has the form

$$
f(x, t)=a_{1} e^{i K_{1}(t) x}+a_{2} e^{i K_{2}(t) x},
$$

with

$$
K_{1}=k_{1} \sin (\omega t), \quad K_{2}=k_{2} \sin (2 \omega t+\theta) .
$$

Consider first the single-harmonic case, $a_{2}=0$. When $t \gg$ $t_{\mathrm{tr}}=1 / \beta$, the soliton oscillations are locked to the driving frequency $\omega$ and are independent of the initial conditions (see Sec. IV). Thus there exists a global solitonic attractor.

We now perform a symmetry analysis $[12,48]$. The perturbed NLS equation is invariant under the symmetry operation

$$
\mathcal{S}: t \mapsto t+T / 2, \quad x \mapsto-x .
$$

At the same time, the transformation $\mathcal{S}$ changes the sign of the soliton velocity $v(t)=\dot{X}(t)$. The soliton position is defined by

$$
X(t)=\frac{\int_{-\infty}^{+\infty} d x x \rho(x, t)}{\int_{-\infty}^{+\infty} d x \rho(x, t)},
$$

with

$$
\rho(x, t)=\left.|| u(x, t)\right|^{2}-\left|u_{\mathrm{bg}}(x, t)\right|^{2} \mid .
$$

Here $u_{\mathrm{bg}}(x, t)=a_{\mathrm{bg}}(t) \exp \left(i K_{1} x\right)$ is the background field to which the soliton decays as $|x| \rightarrow \infty[10,12]$. When $|u(x, t)|^{2}$ from the simulations is plotted, the soliton sits on a shelf with a homogeneous intensity $\left|a_{\mathrm{bg}}(t)\right|^{2}$. The shelf has little influence on the soliton dynamics [10]; this is why the collective coordinate theory is in a good agreement with simulations, despite ignoring the presence of the background.

Since the attractor is global, the transformation $\mathcal{S}$ maps it onto itself. This implies that the average velocity on the attractor is zero. The soliton performs periodic oscillations about its equilibrium position, which are reproduced by the collective coordinate theory [Fig. 7(b)].

In order to construct a solitonic ratchet, i.e., obtain a stable soliton with $\bar{v} \neq 0$, it is necessary to break the invariance under the operation $\mathcal{S}$. The simplest way to do this is to employ the biharmonic driving in Eq. (30) with $a_{1} \neq 0$ and $a_{2} \neq 0$. The collective coordinate equations (9)-(12) can easily be extended to the case of the forcing function $f$ including two terms. In particular, Eq. (9) is replaced with

$$
\dot{\eta}=-2 \beta \eta-\sum_{i=1}^{2} a_{i} \frac{\pi}{2} \operatorname{sech} A_{i} \cos B_{i},
$$

where

$$
\begin{gathered}
A_{i}=\frac{\pi}{4 \eta(t)}\left[K_{i}(t)-p(t)\right], \\
B_{i}=\Phi(t)+K_{i}(t) q(t),
\end{gathered}
$$

while the $K_{i}$ are as in Eqs. (31). The collective coordinate equations for $\dot{q}, \dot{p}$, and $\dot{\Phi}$ are modified in a similar way.

Since the collective coordinate description is accurate only for small perturbations, we take small driving amplitudes $a_{1}=a_{2}=0.05$. We choose a very small driving frequency, $\omega=0.002$, in order to remain in the adiabatic regime. If the damping coefficient $\beta$ is chosen to be too large, the soliton amplitude $\eta$ quickly relaxes to zero, while $q(t)$ and $\Phi(t)$ rapidly go to infinity. For example, for the parameters $\delta=$ $-3, k_{1}=k_{2}=k=0.001, \theta=0$, and the initial conditions $\eta_{0}=1, q_{0}=p_{0}=0, \Phi_{0}=\pi / 2$, this instability occurs when $\beta>0.065$. On the other hand, if $\beta$ is chosen too small (e.g., $\beta=0.01$ ), the average soliton velocity grows without bound over sufficiently long integration times $\left(t_{f} \sim 10^{5}\right)$. Thus we can expect a stable ratchet effect only for intermediate values 
of $\beta$, for instance, $\beta=0.04$. As we find that $\bar{v} \sim k$, a larger ratchet effect can be obtained by increasing $k$. However, when $k$ exceeds a certain critical value $k_{c}$, the average velocity starts to grow slowly with time. (For the chosen parameter values, $\left.k_{c}=0.002\right)$.

Using the parameter values and initial conditions for which the collective coordinate equations exhibit stable solutions, we perform direct simulations of Eq. (1). Our aim is to test whether an initial wave form (8) will evolve into a stable solitary wave over the time $t_{\mathrm{tr}}=1 / \beta$. However, it turns out that the initial structure evolves rapidly immediately after the start of the simulation and quickly decays to zero.

In order to obtain stable solitary waves, we need to improve the initial conditions. This can be achieved by setting the initial conditions equal to the mean values about which the collective coordinates oscillate, once the transients have elapsed. These mean values can be obtained from an approximate analytical solution of the collective coordinate equations. We let

$$
\begin{aligned}
& q=\bar{v} t+C_{q}, \quad p=\bar{p}+C_{\xi}, \\
& \eta=\bar{\eta}+C_{\eta}, \quad \Phi=\bar{\Phi}+C_{\Phi},
\end{aligned}
$$

where $C_{x}$ represent oscillations with amplitude $a_{x}$ and zero mean. We choose $\omega=O\left(10^{-3}\right)$, and $k_{1}=k_{2}=k=O\left(10^{-3}\right)$. The other parameters $\left(\delta, a_{1}, a_{2}, \beta\right)$ do not have to be small for the perturbation analysis that follows and can therefore be chosen to be $O(1)$. By substituting in the collective coordinate equations, we retain only the leading terms in the perturbation series. This gives

$$
\begin{gathered}
\bar{p}=0, \quad p=\frac{a_{1} K_{1}(t)+a_{2} K_{2}(t)}{\left(a_{1}+a_{2}\right)}=O\left(10^{-3}\right), \\
\bar{\eta}=\frac{1}{2} \sqrt{-\delta}, \quad a_{\eta}=O\left(10^{-6}\right), \\
\bar{\Phi}=\arccos \left[\frac{-4 \beta \bar{\eta}}{\pi\left(a_{1}+a_{2}\right)}\right], \quad a_{\Phi}=O\left(10^{-3}\right), \\
q=\bar{v} t+\frac{k}{\omega} \frac{2 a_{1}}{a_{1}+a_{2}}[1-\cos (\omega t)]+\frac{k}{2 \omega} \frac{2 a_{2}}{a_{1}+a_{2}} \\
\times[1-\cos (2 \omega t+\theta)], \quad \bar{v}=O\left(10^{-6}\right) .
\end{gathered}
$$

Equations (39)-(42) are in a very good agreement with the numerical solution of the collective coordinate equations. We note that the constants $\bar{p}, \bar{\eta}$, and $\bar{\Phi}$ do not depend on the relative phase $\theta$, but the variable components of $q(t)$ and $p(t)$ do.

The improved initial conditions are now $p_{0}=\bar{p}=0, \eta_{0}=$ $\bar{\eta}, \Phi_{0}=\bar{\Phi}$, and $q_{0}=0$. After a transient time, the numerical trajectory settles to the solution (39)-(42). This yields $v(t)=$ $\dot{q}=2 p$, and thus $p(v)=\frac{1}{2} v$ is a straight line with positive slope. Our stability criterion predicts the stability of the soliton and simulations of the PDE confirm this [Figs. 8(a) and 8(b)]. As $\bar{v}$ is very small, the ratchet effect is not visible on the time scale of Figs. 8(a) and 8(b), but can be observed over longer simulation times $t_{f} \approx 30 T_{d}$ [Fig. 8(c)]. The soliton collective coordinate $\eta(t)$ oscillates about 0.866025 in the interval [0.866024,0.866026], which is in agreement with Eq. (40). In the simulations, $\eta(t)$ oscillates about 0.87363 in the interval [0.87 305,0.87 390].

The ratchet effect is also observed for higher driving frequencies (e.g., $\omega=0.01$ and 0.02). However, as $\omega$ is
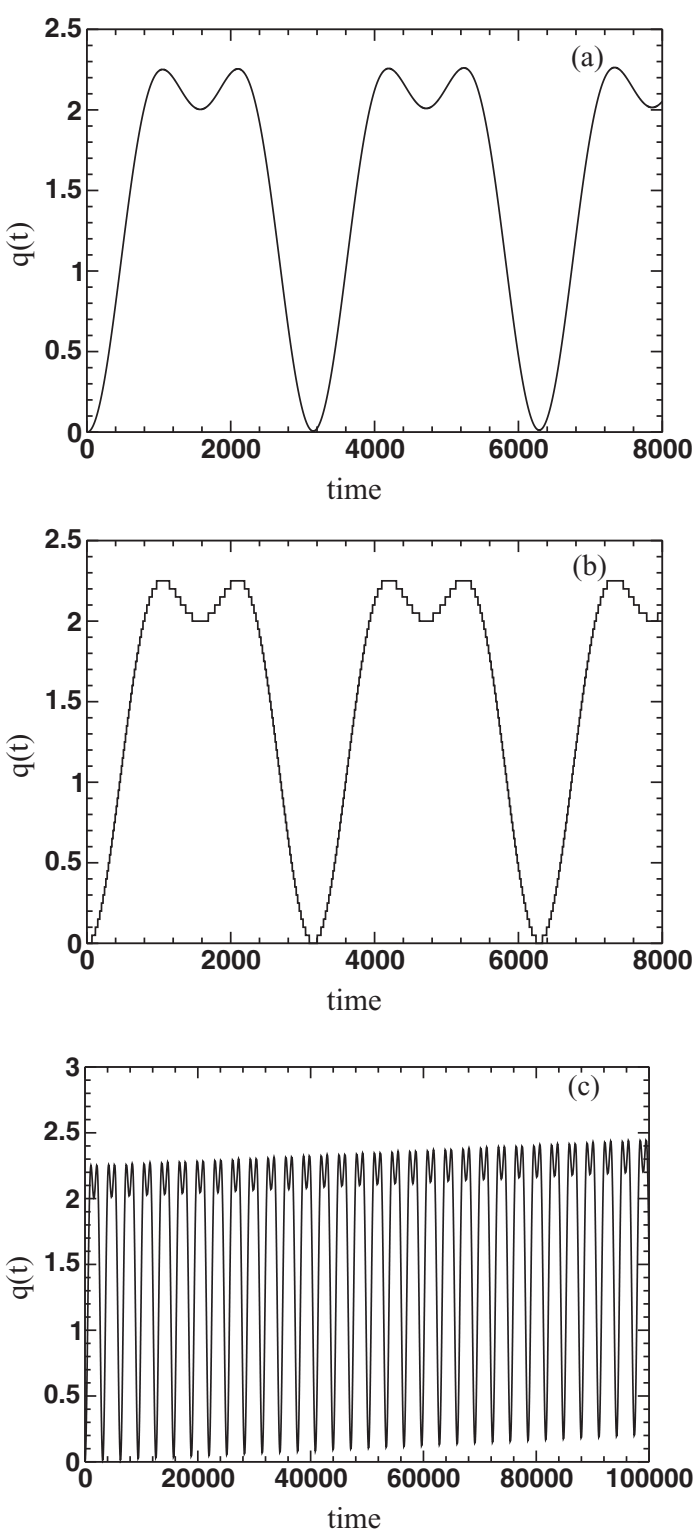

FIG. 8. Soliton position $q(t)$ : (a) from the collective coordinate theory, (b) from simulations, and (c) the ratchet effect in the collective coordinate theory visible over long times ( $t_{f} \approx 30$ periods). Parameters: $a_{1}=a_{2}=a=0.05, k_{1}=k_{2}=k=0.002, \delta=-3, \omega=0.002$, $\theta=0$, and $\beta=0.08$, with initial conditions $\eta_{0}=\bar{\eta}, q_{0}=p_{0}=0$, $\Phi_{0}=\bar{\Phi}$

increased, both $\bar{v}$ and the last two terms in Eq. (42) decay in proportion to $1 / \omega$. Therefore, both the instantaneous and the average velocity become small and difficult to detect when $\omega$ is large.

It is important to emphasize here that our ability to determine the initial conditions leading to stable solitons is due to the availability of the explicit solution of the collective coordinate equations and our stability criterion. It would be very difficult to localize the corresponding small basin of attraction by scanning the parameters of the initial conditions in the numerical simulations of the PDE.

Finally, we discuss the dependence of the average velocity $\bar{v}$ on the relative phase $\theta$ in the biharmonic driving force (31). 
As expected for a ratchet system with a biharmonic driving [48,49], $\bar{v}(\theta)$ is sinusoidal with the period $2 \pi$. It attains its maximum value near $\theta=0$ and its small negative minimum value near $\theta=\pi$. The size and shape of the basin of attraction around $(\bar{\eta}, \bar{\Phi})$ also depend strongly on $\theta$; this effect will be examined in a future work.

\section{SUMMARY}

We have formulated a refined empirical stability criterion for the driven NLS solitons. Unlike stability criteria available in the literature, the new criterion is based on a collective coordinate (CC) description. Solving (analytically or numerically) evolution equations for the four collective coordinates, we use the resulting trajectories to evaluate the normalized soliton momentum $p(t)$ and the soliton velocity $v(t)$. These give a parametric "stability curve," $p(v)$.

Whenever the curve $p(v)$ has a section with a negative slope $(d p / d v<0)$, we observe the instability of the soliton in direct numerical simulations. We, therefore, conjecture that the availability of a section with a negative slope is a sufficient condition for the instability of the soliton. We do not have a mathematical proof of this conjectured criterion; however, we have verified it in a variety of situations using constant, harmonic, and biharmonic functions $K(t)$, with or without the damping term.

The establishment of a theoretical justification for this conjecture is a subject for future work, first for the cases of $K(t)$ which we have considered in this paper, then for a general function $K(t)$. One of the foreseen difficulties is related to the fact that the soliton solution of the driven NLS equation does not vanish as $x \rightarrow \pm \infty$ because the perturbation $f(x, t)=a \exp [i K(t) x]$ does not decay to zero in these limits. On the other hand, our collective coordinate theory is based on a soliton ansatz which vanishes as $x \rightarrow$ $\pm \infty$.

For the case of constant $K$ and zero damping, all collective coordinates perform periodic motions. This allowed us to compute a phase portrait, which consists of closed orbits on a complex plane. The soliton evolution is described by the motion along one of these orbits. We observe that the sense of rotation of the orbit is correlated with the stability/instability of the soliton (determined in simulations of the full PDE). Namely,

(i) if the orbit is an ellipse with a positive sense of rotation, the soliton is stable;

(ii) if the orbit is a horseshoe where the inner part has a negative and the outer part a positive sense of rotation, the soliton is unstable and disintegrates very quickly; and

(iii) if the orbit is an ellipse with a negative sense of rotation, then the soliton remains metastable for a relatively long time, but eventually disintegrates.

An interesting question is whether our collective coordinate approach and stability criteria can be applied to perturbed NLS equations with a more general form of the nonlinearity. Work is in progress regarding the general power-law nonlinearity, $\left(u^{*} u\right)^{\kappa}$, where $\kappa=1$ corresponds to the NLS equation of this paper. The underlying unperturbed NLS equation has stable solitons for $0<\kappa<2$, and it will be interesting to determine how the stability of the solitons is affected by the perturbation $f(x, t)$.

\section{ACKNOWLEDGMENTS}

F.G.M. acknowledges the hospitality of the Mathematical Institute of the University of Seville (IMUS) and of the Theoretical Division and Center for Nonlinear Studies at the Los Alamos National Laboratory. Work at Los Alamos was supported by the US Department of Energy. F.G.M. acknowledges financial support by the Plan Propio of the University of Seville and by Junta de Andalucía under Grant No. IAC09-III-6399. N.R.Q. acknowledges financial support by the DAAD under Grant No. A/08/04067, by the Ministerio de Educación y Ciencia (MEC, Spain) through Project No. FIS2008-02380/FIS, and by Junta de Andalucía under Projects No. FQM207, No. FQM-00481, No. P06-FQM-01735, and No. P09-FQM-4643.

\section{APPENDIX: FIXED POINTS OF THE PHASE PORTRAIT}

For the case of the time-independent force $f(x)=a e^{i K x}$ and zero damping, we adopt the following ansatz for stationary solutions of the collective coordinate equations:

$$
q(t)=v_{s} t, \quad \eta(t)=\eta_{s}, \quad p(t)=p_{s}, \quad \Phi(t)=\Phi_{s}-\alpha_{s} t .
$$

Equation (9) yields $\cos B \equiv 0$, which results in

$$
K v_{s}=\alpha_{s}, \quad \Phi_{s}^{ \pm}= \pm \frac{\pi}{2}, \quad \sin B= \pm 1 .
$$

We insert the ansatz (A1) in Eq. (26), yielding

$$
\Psi_{s}=2 i \eta_{s} \operatorname{sech}\left[2 \eta_{s}\left(\bar{v}-v_{s}\right) t\right] e^{-i\left[-p_{s}\left(\bar{v}-v_{s}\right) t+\Phi_{s}+\left(K \bar{v}-\alpha_{s}\right) t\right]} .
$$

The fixed points of the phase portrait correspond to the timeindependent $\Psi$, i.e., $\bar{v}=v_{s}$. By using Eqs. (A2), we obtain two fixed points

$$
\operatorname{Re} \Psi_{s}^{ \pm}= \pm 2 \eta_{s}, \quad \operatorname{Im} \Psi_{s}^{ \pm}=0
$$

The \pm signs refer to the two cases in Eqs. (A2).

Combining Eqs. (10) and (12) with Eq. (A2), $\alpha_{s}$ can be eliminated and we are left with two equations,

$$
\begin{gathered}
v_{s}=2 p_{s} \pm \frac{a \pi^{2}}{8 \eta_{s}^{2}} \operatorname{sech} A_{s} \tanh A_{s}, \\
-\left(K-p_{s}\right) v_{s}=p_{s}^{2}-4 \eta_{s}^{2}-\delta \pm \frac{a \pi}{2 \eta_{s}} A_{s} \operatorname{sech} A_{s} \tanh A_{s},
\end{gathered}
$$

where $A_{s}=\pi\left(K-p_{s}\right) /\left(4 \eta_{s}\right)$. For either sign, the system (A5)-(A6) has a single root $v_{s}=2 p_{s}, p_{s}=K$, and $\eta_{s}=$ $\frac{1}{2} \sqrt{K^{2}-\delta}$. Therefore, there are two fixed points on the real axis, located at $\operatorname{Re} \Psi= \pm \sqrt{K^{2}-\delta}$.

For the set of parameters of Fig. 2, there is a stable and an unstable fixed point close to +1 and -1 , respectively. The stability of the fixed points is determined by solving the collective coordinate equations numerically with the initial conditions very close to the above values, e.g., $\eta_{0}=\eta_{s}+10^{-8}$, $q_{0}=0, p_{0}=p_{s}$, and $\Phi_{0}=\Phi_{s}^{ \pm}= \pm \pi / 2$. In the unstable case 
$\left(\Phi_{0}=-\pi / 2\right)$, the numerical solution exhibits oscillations of the amplitude and phase, whereas the velocity of the soliton remains constant. This solution is represented by the separatrix in Fig. 2. A trivial stable fixed point is located at the origin; its stability is established by numerical solutions of the collective coordinate equations with $\eta_{s}$ close to zero.
[1] D. J. Kaup and A. C. Newell, Proc. R. Soc. London A 361, 413 (1978); Phys. Rev. B 18, 5162 (1978).

[2] P. S. Lomdahl and M. R. Samuelsen, Phys. Rev. A 34, 664 (1986).

[3] A. W. Snyder and J. D. Love, Optical Waveguide Theory (Chapman and Hall, London, 1983).

[4] B. A. Malomed, Phys. Rev. E 51, R864 (1995).

[5] G. Cohen, Phys. Rev. E 61, 874 (2000).

[6] K. Nozaki and N. Bekki, Physica D 21, 381 (1986).

[7] I. V. Barashenkov and Y. S. Smirnov, Phys. Rev. E 54, 5707 (1996).

[8] I. V. Barashenkov and E. V. Zemlyanaya, Physica D 132, 363 (1999).

[9] V. M. Vyas, T. S. Raju, C. N. Kumar, and P. K. Panigrahi, J. Phys. A 39, 9151 (2006).

[10] F. G. Mertens, N. R. Quintero, and A. R. Bishop, Phys. Rev. E 81, 016608 (2010).

[11] U. Peschel, O. Egorov, and F. Lederer, Opt. Lett. 15, 1909 (2004).

[12] A. Gorbach, S. Denisov, and S. Flach, Opt. Lett. 31, 1702 (2006).

[13] D. Poletti, E. A. Ostrovskaya, T. J. Alexander, B. Li, and Y. S. Kivshar, Physica D 38, 1338 (2009).

[14] M. Rietmann, R. Carretero-González, and R. Chacón, Phys. Rev. A 83, 053617 (2011).

[15] M. Salerno and Y. Zolotaryuk, Phys. Rev. E 65, 056603 (2002).

[16] L. Morales-Molina, N. R. Quintero, F. G. Mertens, and A. Sánchez, Phys. Rev. Lett. 91, 234102 (2003).

[17] A. V. Ustinov, C. Coqui, A. Kemp, Y. Zolotaryuk, and M. Salerno, Phys. Rev. Lett. 93, 087001 (2004).

[18] L. Morales-Molina, F. G. Mertens, and A. Sánchez, Phys. Rev. E 72, 016612 (2005).

[19] P. Müller, F. G. Mertens, and A. R. Bishop, Phys. Rev. E 79, 016207 (2009).

[20] R. Scharf and A. R. Bishop, Phys. Rev. E 47, 1375 (1993).

[21] N. G. Vakhitov and A. A. Kolokolov, Radiophys. Quantum Electron. 16, 783 (1975).

[22] M. I. Weinstein, Commun. Pure Appl. Math. 39, 51 (1986).

[23] Y. Sivan, G. Fibich, B. Ilan, and M. I. Weinstein, Phys. Rev. E 78, 046602 (2008).

[24] N. Akhmediev, A. Ankiewicz, and R. Grimshaw, Phys. Rev. E 59, 6088 (1999).

[25] M. M. Bogdan, A. S. Kovalev, and A. M. Kosevich, Sov. J. Low Temp. Phys. 15, 288 (1989).

[26] I. V. Barashenkov and E. Yu. Panova, Physica D 69, 114 (1993).
[27] D. E. Pelinovsky, Y. S. Kivshar, and V. V. Afanasjev, Phys. Rev. E 54, 2015 (1996).

[28] I. V. Barashenkov, Phys. Rev. Lett. 77, 1193 (1996).

[29] I. V. Barashenkov, E. V. Zemlyanaya, and M. Bär, Phys. Rev. E 64, 016603 (2001).

[30] Y. Kivshar and B. Malomed, Rev. Mod. Phys. 61, 763 (1989).

[31] W. L. Kath, Methods Appl. Analysis 4, 141 (1997).

[32] N. R. Quintero, F. G. Mertens, and A. R. Bishop, Phys. Rev. E 82, 016606 (2010).

[33] I. V. Barashenkov, Y. S. Smirnov, and N. V. Alexeeva, Phys. Rev. E 57, 2350 (1998).

[34] I. V. Barashenkov, T. Zhanlav, and M. M. Bogdan, in Nonlinear World, Proceedings of the Fourth International Workshop on Nonlinear and Turbulent Processes in Physics, Singapore, 1990, edited by V. G. Bar'yakhtar et al. (World Scientific, Singapore, 1990), pp. 3-9.

[35] P. Hänggi and R. Bartussek, in Nonlinear Physics of Complex Systems-Current Status and Future Trends, edited by J. Parisi et al., Lecture Notes in Physics, Vol. 746 (Springer, Berlin, 1996), p. 476.

[36] R. D. Astumian and P. Hänggi, Phys. Today 55, 33 (2002).

[37] P. Hänggi, F. Marchesoni, and F. Nori, Ann. Phys. (Leipzig) 14, 51 (2005).

[38] F. Jülicher, A. Ajdari, and J. Prost, Rev. Mod. Phys. 69, 1269 (1997).

[39] P. Reimann, Phys. Rep. 361, 57 (2002).

[40] Ratchets and Brownian Motors: Basics, Experiments and Applications, edited by H. Linke, special issue of Appl. Phys. A 75, 2 (2002).

[41] I. Zapata, R. Bartussek, F. Sols, and P. Hänggi, Phys. Rev. Lett. 77, 2292 (1996).

[42] F. Marchesoni, Phys. Rev. Lett. 77, 2364 (1996).

[43] F. Falo, P. J. Martínez, J. J. Mazo, and S. Cilla, Europhys. Lett. 45, 700 (1999).

[44] E. Trías, J. J. Mazo, F. Falo, and T. P. Orlando, Phys. Rev. E 61, 2257 (2000).

[45] M. Salerno and N. R. Quintero, Phys. Rev. E 65, 025602(R) (2002).

[46] G. Costantini, F. Marchesoni, and M. Borromeo, Phys. Rev. E 65, 051103 (2002).

[47] A. V. Gorbach, S. Denisov, and S. Flach, Chaos 16, 023125 (2006).

[48] S. Flach, Y. Zolotaryuk, A. E. Miroshnichenko, and M. V. Fistul, Phys. Rev. Lett. 88, 184101 (2002).

[49] L. Morales-Molina, N. R. Quintero, A. Sánchez, and F. G. Mertens, Chaos 16, 013117 (2006). 